\title{
A Proposal for a Los Alamos International Facility for Transmutations (LIFT)
}

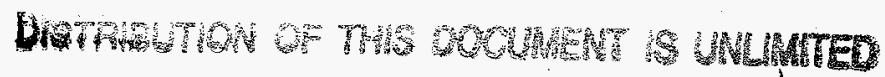

im

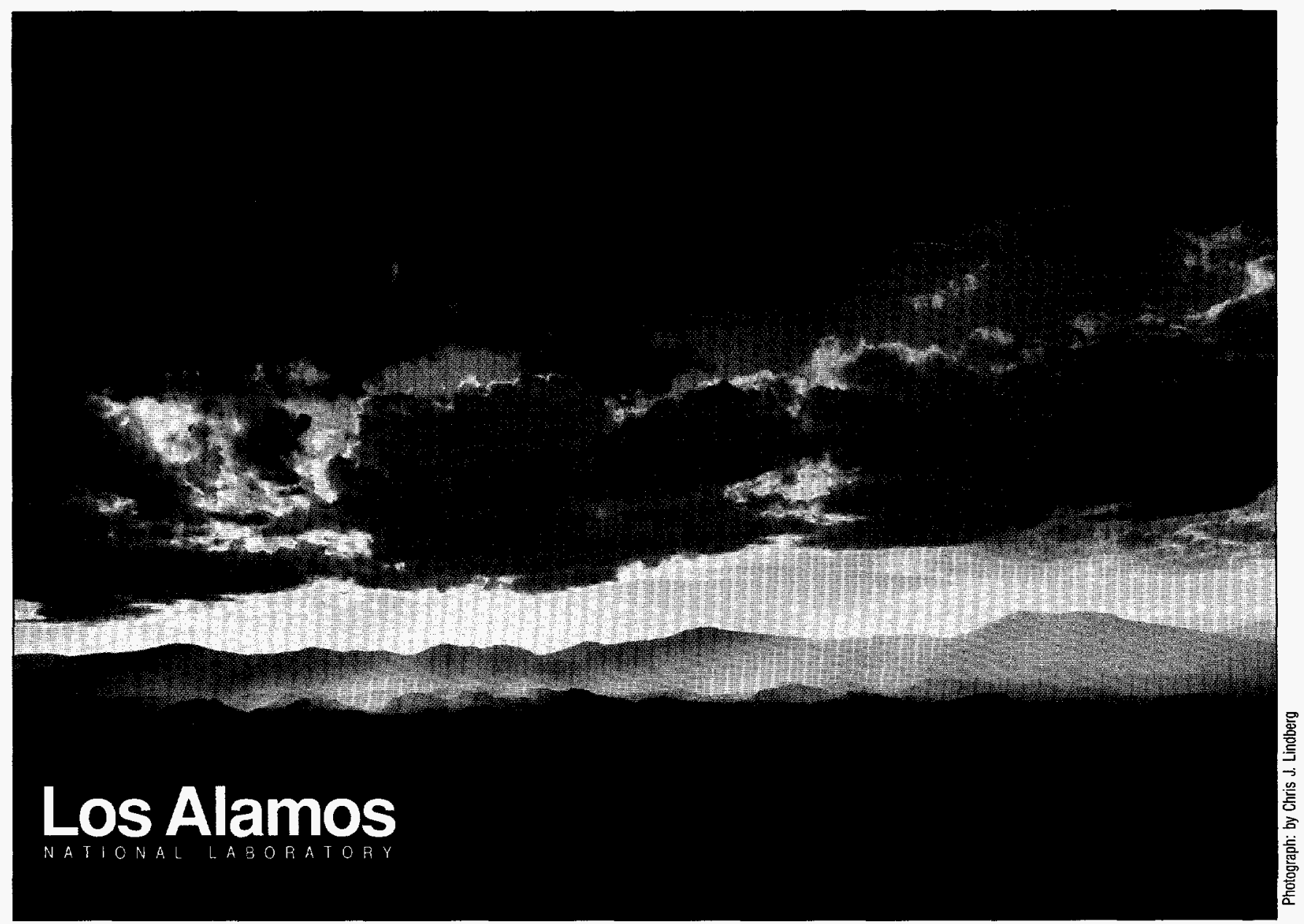

This is a preprint of a paper intended for publication in a journal or proceedings. Because changes may be made before publication, this preprint is made available with the understanding that it will not be cited or reproduced without the permission of the author. 


\section{DISCLAIMER}

This report was prepared as an account of work sponsored by an agency of the United States Government. Neither the United States Government nor any agency thereof, nor any of their employees, make any warranty, express or implied, or assumes any legal liability or responsibility for the accuracy, completeness, or usefulness of any information, apparatus, product, or process disclosed, or represents that its use would not infringe privately owned rights. Reference herein to any specific commercial product, process, or service by trade name, trademark, manufacturer, or otherwise does not necessarily constitute or imply its endorsement, recommendation, or favoring by the United States Government or any agency thereof. The views and opinions of authors expressed herein do not necessarily state or reflect those of the United States Government or any agency thereof. 


\section{DISCLAMMER}

Portions of this document may be illegible in electronic image products. Images are produced from the best available original document. 
LA-UR- 96-3567

Approved for public release distribution is unlimited.
Title:

Author(s):

Submitted to:
A Proposal for a Los Alamos International Facility for Transmutations (LIFT)

Francesco Venneri, LER/ADTT Mark A. Williamson, NMT-6

Ning Li, MST-10

Gary Doolen, LER/ADTT

\section{Los Alamos}

NATIONAL LABORATORY

Los Alamos National Laboratory, an affirmative action/equal opportunity employer, is operated by the University of California for the U.S. Department of Energy under contract W-7405-ENG-36. By acceptance of this article, the publisher recognizes that the U.S. Government retains a nonexclusive, royalty-free license to publish or reproduce the published torm of this contribution, or to allow others to do so, for U.S. Government purposes. Los Alamos National Laboratory requests that the publisher identify this arlicle as work performed under the auspices of the U.S. Department of Energy. The Los Alamos National Laboratory strongly supports academic freedom and a researcher's right to publish; as an institution, however, the Laboratory does not endorse the viewpoint of a publication or guarantee its technical correctness. 


\section{A Proposal for a \\ Los Alamos International Facility for Transmutations (LIFT)}

Francesco Venneri, Mark Williamson, Ning Li, Gary Doolen LER-ADTT Los Alamos National Laboratory

Los Alamos 11/22/96

\section{Summary}

The thrust of the ATW concept ....

The following points have been established ....

Based on the preceding considerations ....

ATW Experimental Program

LIFT, an International Facility for Transmutations at Los Alamos

LIFT Strategy 


\section{Summary:}

\section{Motivation}

The major groups engaged in transmutation research are converging towards a common objective and similar technology. It is now possible to envision an international program of research aimed at the destruction of reactor-generated (and other) nuclear waste using a series of multipurpose experimental facilities in the near future. Los Alamos National Laboratory, as the home of the highest power LINAC and a very active transmutation technology project, is the ideal host for the first of such facilities. The next step in the international program (a facility 10 times more powerful, for engineering-scale demonstrations) could be built in Europe, where there is substantial interest in the construction of such a device in the framework of international cooperation.

\section{Idea Outline}

At Los Alamos: A series of experiments exploring the key transmutation technologies. Liquid lead loops, a liquid lead spallation target, and a large size liquid lead facility with provision for irradiation, cooling and diagnostics of several types of "transmutation assemblies", where different transmutation concepts will be tested in different media and environments, from transmutation of fission products to destruction by fission of higher actinides, to other waste management applications. The engineering-scale facility, which will follow the initial testing phase, will extend the best concepts to full scale implementation.

\section{Interested Parties}

US Laboratories (LANL, ANL, BNL, LLNL, ORNL, SNL).

US Universities (UC Berkeley, Illinois, MIT)

DOE-EM, DOE-ER, DOE-OCRWM (Stake holders)

US Industry (Grumman, Bechtel, General Atomics, Westinghouse, Rockwell, TRW, Lockheed)

CERN, ENEA, INFN, CEA, JAERI, KAERI, Sweden, Czech Rep., Russia (Obninsk-IPPE, Chelyabinsk)

\section{Time Line}

1997 LANL

1999 LANL

2001 LANL

2006 Europe

2010 US / Europe
Liquid lead loops

Obninsk-IPPE target

Liquid Lead Multipurpose Transmutation Exp. Engineering-scale Transmutation Facility (Next Step)

Simultaneous deployment of full-scale ATW
LIFT-Stage I

LIFT- Stage II

LIFT- Stage III

\section{Options for Funding}

To be discussed

\section{Immediate Next Steps}

Raise issue of International Transmutation Facility to government officials and interested Laboratories, Universities and Industry. Develop international and US support for the facility. Coordinate plan of action with all interested parties.

If there is sufficient national and international interest, Los Alamos will be very enthusiastic to entertain the siting of the transmutation test facility at LANSCE. 


\section{The thrust of the ATW concept lies in the novel combination of particle accelerators and nuclear reactor technology.}

Development of high-current accelerators suitable for ATW is presently funded in APT (the Accelerator Production of Tritium project) . Based on the projections and progress of that program, we can reasonably assume that the technology will be available in the relevant time frame to ATW development. We need to monitor the development periodically, but no independent effort will be expended on this aspect.

The target/blanket configuration is the key component of ATW. To maximize its unique benefits, ATW should be based on the best reactor technology. Although aqueous thermal reactors and the associated PUREX reprocessing represent the most mature technology, fast metal reactors are far superior in terms of neutron economy, subcritical moderated nuclear assemblies offer lower-inventory advantages and dry chemical processes for fuel preparation and cleanup are acknowledged to be potentially much less expensive and complex. These are the foundations we intend to build on in developing the ATW system at Los Alamos.

The accelerator-driven subcritical assembly of ATW allows for neutronic advantage, flexibility and robustness of operations not comparably available in critical reactors. Therefore, fission/transmutation optimization of the subcritical assembly should be the first order of research activity. Because of its role, the neutronics study should be the foremost focused area of research. Work is now being done aggressively to optimize the neutron spectra for fission and transmutation use. Since the relevant chemical processes for most possible configurations (molten salts, liquid metals; solid fuel and liquid fuel, etc) do exist in some forms (ORNL processes for molten fluoride salts, modified at LANL ADTT; ANL-LANL metallurgical processes for IFR; BNL and LANL processes for liquid bismuth fuels), the principal effort will be expended on searching out the common threads of these technologies and further developing them, eventually merging them with the system's neutronics.

Progress in the analysis has been rapid, and the need is emerging for a significant experimental effort to substantiate the advantages of subcritical operation and select the best solutions for waste destruction. 


\section{The following points have been established, and can now be considered part of the Los Alamos ATW base.}

1.) If ATW is to be a worthy technological alternative to a critical reactor, it should provide objective and substantial advantages over the best reactor concepts in the treatment of nuclear waste, such as: larger number of available neutrons per fission, greater fuel flexibility, more rugged operation.

2.) Fast spectrum systems have a very large neutronic advantage compared to thermal systems, which can be fully exploited in subcritical configurations. On the order of 10 times more available neutrons per fission can be utilized by subcritical fast systems with the accelerator size as in thermal systems.

3.) The total actinide inventory in any nuclear system is proportional to the cross section, the flux, process volumes (including heat exchangers and ducts), and the decontamination factor required. While the fission and capture cross sections of fissionable isotopes, such as plutonium 239 and 241 are lower by two orders of magnitude going from thermal to fast, the cross sections of the real "bottlenecks" (the actinide isotopes that do not fission in the thermal spectrum, such as Cm-244 and Cm248) are only lower by a factor of 5 . Because of the unfavorable ratio of capture-tofission cross sections for thermal neutrons, the higher actinides pile-up and $\mathrm{Cm}-244$ and $\mathrm{Cm}-248$ eventually become the most abundant actinide isotopes in a thermal spectrum after protracted burn. Once the larger neutron flux of fast systems (factor of 10) and their lower process volumes and decontamination requirements (factor of 2-4) are taken into account, the total actinide inventory in properly designed subcritical fastspectrum systems becomes comparable to those of thermal systems after protracted burn periods. Finally, a fast-spectrum burn cycle followed by thermalization and burn-down of inventories should be feasible in subcritical liquid-fueled systems and will offer substantial end-of-life inventory advantages with respect to a protracted fully thermal burn, including the avoidance of buildups of the very high actinides ( $\mathrm{Am}, \mathrm{Cm}, \mathrm{Bk}, \mathrm{Cf})$.

4.) Thermohydraulics. Inert, heavyweight liquid metals are the preferred 
media for ATW in terms of thermohydraulics. Inert liquid metal nuclear technology (lead, lead/bismuth and lead/lithium) is well developed in Russia, less so in the US. Liquid lead is the best available material for spallation neutron production for ATW applications. ATW will take advantage of the lead technology as much as possible, and uses it as both target and coolant medium.

\section{5.) Process Chemistry. Molten-salt and liquid-metal based processes are} the preferred options for process chemistry. We have developed molten-salt and liquid-metal process flow sheets that include front-end fuel preparation from spent fuel and back-end cleanup. Proliferation-resistance of the processes has been and will remain the most important driver in the technology development.

6.) Dilution is not acceptable as a means to meet low-level waste specification for the final waste stream. Ultimate disposition of ATW waste will require some storage in permanent repository. ATW treatment of nuclear waste very positively affects repository performance not only in terms of large mass and volume reduction (elimination of actinides and key fission products), but also in the qualitative change of the resultant waste forms (fission product partitioning). The optimal location of ATW plants is at the site of final waste storage.

7.) ATW is envisioned as a centralized facility, with appropriate oversight and protection against proliferation. ATW will treat spent fuel and nuclear waste using processes that do not produce pure streams of plutonium or other weapons grade materials, but need to be thoroughly monitored nonetheless. The value and feasibility of ATW depends crucially on the overall strategy of nuclear waste management.

\section{8.) ATW's essential attributes are availability of neutrons and flexibility} of operations above and beyond those of a critical nuclear reactor. These come at a price premium (the cost of the accelerator). ATW therefore is not a replacement for energy-producing nuclear power reactors. The role of ATW is in the disposition of objectionable or unwanted nuclear material that, for any number of reasons (technical and otherwise), cannot be destroyed in reactors or disposed in unattended permanent storage sites. 


\section{Based on the preceding considerations, a new concept for ATW is being assessed at Los Alamos, whose main features are:}

1. Liquid lead (or lead/bismuth), used as a target material and as a coolant for the fuel

2. Molten salt, used as a fuel and carrier for the process chemistry

3. Liquid bismuth, used as a fuel and carrier for the process chemistry

4. Fast spectrum operation with final thermal burn-down of inventories

The Transmutation System layout consists of a large liquid lead enclosed volume which includes a central target with surrounding submerged transmutation assemblies, containing actinide and fission product solutions. Also possible are breeding assemblies containing thorium and uranium. Liquid metal and molten salt assemblies are possible, containing fission products and/or actinides dissolved in a suitable medium (e.g. bismuth or fluorides). Fuel chemistry has been identified for both salt and metal fuel forms and is actively being researched. The assemblies can be either sealed or open to the processing volume, and the liquid fuel could be either stagnating or slowly moving to and from processing in a semi-continuous fashion. The assemblies are all cooled by the flowing liquid lead. Successful implementation of the "liquid fuel in a can" approach could eventually lead to a one-fluid system. At this stage however we are not considering this option actively.

For best neutronic performance, the neutron spectrum is fast for the majority of operating life of the plant, and is thermalized at the end of life by termination of the actinide feed in the fuel loops and gradual introduction of moderator in the lead coolant. Thermalizers, such as beryllium, can be used in the fission product transmutation assemblies. The fast spectrum destruction of the actinides will allow full utilization of the neutrons generated from the fission of the higher actinides in the fuel, avoiding the buildup of very high actinides ( $\mathrm{Am}, \mathrm{Cm}, \mathrm{Bk}, \mathrm{Cf}$ ). Considerable effort will be spent in the design of the "thermal" neutron traps to be used for transmutation of fission products.

Both low-multiplication and high-multiplication subcritical systems are being considered, as they have different operational regimes, neutronic parameters and possible applications. After the thermalized burn-down phase, end-of-life inventories can be reduced to levels much lower than what would have been obtained through a continuous thermal burn. 
There are several advantages to this approach:

1.) Compatibility of structural materials with salt and lead is facilitated by the relatively low temperature of the coolant $\left(<400{ }^{\circ} \mathrm{C}\right)$. Good radiation resistance to fast neutrons is available from well tested nuclear structural materials operating around $400^{\circ} \mathrm{C}$.

\section{2.) Liquid lead and lead/bismuth thermohydraulics and components} engineering have been extensively developed. No fission products or actinides will be present in the coolant and intermediate heat exchangers might not be necessary. Spallation products will be very diluted in the large volume of the target lead and will not significantly affect the performance of the ATW system. Process chemistry is being investigated to extract the spallation products from the liquid lead, if needed.

3.) The molten salt or liquid metal fuel assembly, containing slow moving fluid, will behave similarly to solid fuel rods, with the difference that fission products can be extracted and will not build up. The problems associated with material corrosion should be reduced by the low temperature of the salt and metal fuel container walls.

4.) The fast spectrum system will allow full utilization of the neutrons generated from the fission of the higher actinides in the fuel. At the same time the buildup of very high actinides ( $\mathrm{Am}, \mathrm{Cm}, \mathrm{Bk}, \mathrm{Cf}$ ) is avoided. After the thermalization and burn-down phase, inventories are reduced to levels lower than what would have been obtained through a continuous thermal burn. The gradual thermalizing of the spectrum in the burn-down phase will allow the full utilization of resonance absorptions for inventory reduction. 


\section{ATW Experimental Program:}

This succession of experiments will foster the development of the ATW technology and build the expertise necessary for deployment by the year 2010:

1) $1997-1998$

\section{Liquid lead loop construction, operation at LANSCE}

Process chemistry and ATW preconceptual design

2) $1999-2000$

Obninsk-IPPE target tested at LANSCE (OTT). Process chemistry (scale-up) and ATW conceptual design

3) 2001-2005

Liquid Lead Multipurpose Transmutation Experiment (LIFT-1). Construction of a large liquid lead assembly to receive the full LANSCE beam, using the technology obtained in (2), with provision for several transmutation loops, both moderated and unmoderated, cooled by the lead, so that different forms of "fuel forms" would be tested. The "fuel forms" could be solid or liquid, metallic or salt or water based, containing fission products, thermalizing materials and/or actinide surrogates to absorb neutrons and test the performance of several concepts. Fissionable materials will eventually be tested in these loops. Total thermal power will be up to 5 MW. Highe fission power levels are not likely at Los Alamos. Small-scale molten salt and liquid metal cleanup processes will be conducted on the irradiated materials.

4) $2006-2010$

An Engineering-scale Liquid Lead Target/Blanket (LIFT-II) will be built at the site of a future large ( $>10 \mathrm{~mA}$ ) LINAC with provision for full size fuel and transmutation loops.

Essential engineering tests will be performed on the lead target/coolant system.

Large- scale fission product transmutation. Large-scale minor actinide transmutation. Spectrum shift experiments (introduction of moderator in the lead coolant).

LIFT-II, will likely be a fixed-fuel fast subcritical assembly, with multiplication up to 0.95 , with integrated target/coolant liquid lead loop, powered by a large LINAC (10-20 mA, 1-2 GeV). Many locations will be provided for the characterization of actinide and fission product 
transmutation and the testing of transmutation assemblies in high neutron flux. Progressively, more and more of the fixed fuel will be replaced by the transmutation assemblies. Total thermal power will likely be up to $100 \mathrm{MW}$. Europe is the most likely venue for this facility, because: 1.) They are willing to build the ATW-dedicated LINAC. 2.) They are willing to build the fast subcritical facility. Savannah River (SRS) could be an alternate (concurrent?) site in the US, if it became possible to merge ATW and APT at some level. 


\section{LIFT, An International Facility for Transmutations at Los Alamos}

Recent advances in the Science and Technology base of the three major transmutation projects (LANL, CEA, CERN, JAERI) have made possible the formulation of a common approach to the development of transmutation systems. All three leading transmutation technology groups are now focused on liquid metal cooled systems, fast spectrum operation and the destruction of waste actinides. Repeatedly, and most notably at the International ADTT Conference in Kalmar (Sweden, June 1996), the call has been made for a more concerted approach among the international transmutation community, in light of the convergence of interest and approach among the leading programs, and especially in view of the increasing need for moderate and large scale experiments requiring substantial levels of financial commitment.

A large component of International Collaboration is now already present in the Los Alamos transmutation program. Liquid lead/bismuth technology (of paramount importance to the project) is being developed using Russian-US-EC cooperation under the auspices of ISTC. International participation in testing and operation of the IPPE-Obninsk target will occur. Other important components of the Los Alamos ATW technology are also being pursued cooperatively by Russian scientists with ISTC funding from four major international participants (EC, Sweden, Japan, US).

The Obninsk-IPPE large volume liquid lead target test (OTT) and the the LANSCE Multipurpose Transmutation Experiment (LIFT-I), anticipated at Los Alamos for the years 1999-2000 and 2001-2006 respectively, will provide ample opportunity for international participation. The target will provide fundamental liquid lead technology expertise in high-flux proton and neutron environment. The LIFT-I facility (consisting of the large lead target/coolant system with provision for diverse transmutation loops and the addition of adequate heat removal) will provide realistic testing to many different nuclear waste transmutation approaches.

Substantial interest has been building to the idea of a multi user experimental program on transmutations, involving domestic and international participation at Los Alamos, using the unique facilities that could be made available in the near future. 


\section{LIFT Strategy}

In the LIFT scenario, LANSCE would become the host of the first international facility for physics and engineering testing of nuclear waste transmutation concepts. LIFT would provide a common platform, compatible with CERN's, CEA's, JAERI's and most other transmutation approaches. The facility could be ready to perform testing, research and development by the participating parties, using the LANL accelerator, before the year 2000 (on the Obninsk-IPPE target). LIFT would have very significant value to all the participating transmutation groups, without conflicting with their technical paths.

Initial funding for construction and establishment of the facility at Los Alamos should be facilitated by the following considerations: 1) LIFT would be consistent with present DOE and US guidelines on fuel reprocessing, since the facility would not be engaged in fuel reprocessing, but only in transmutations. 2) LIFT would not require the US alone to have a commitment to the technology, because the facility will be part of a sequence of efforts aimed at the development of the technology on an international basis. 3) LIFT would comply to site-wide EIS for the LANSCE facility at Los Alamos.

A long time-scale strategy of sequential experimental facilities dedicated to waste (and other) transmutations could be as follows: 1) Starting at Los Alamos in the immediate future (OTT and LIFT-I), and progressing to a medium size machine in Europe (LIFT-II, using a 10-20 mA accelerator, already being discussed in Italy and elsewhere) and finally to two full size facilities, one in the US and one in Europe, to detoxify the waste generated by reactor operations in the two continents prior to final disposal of the irreducible residues. 


\section{ATW-Assisted Fuel Cycle}

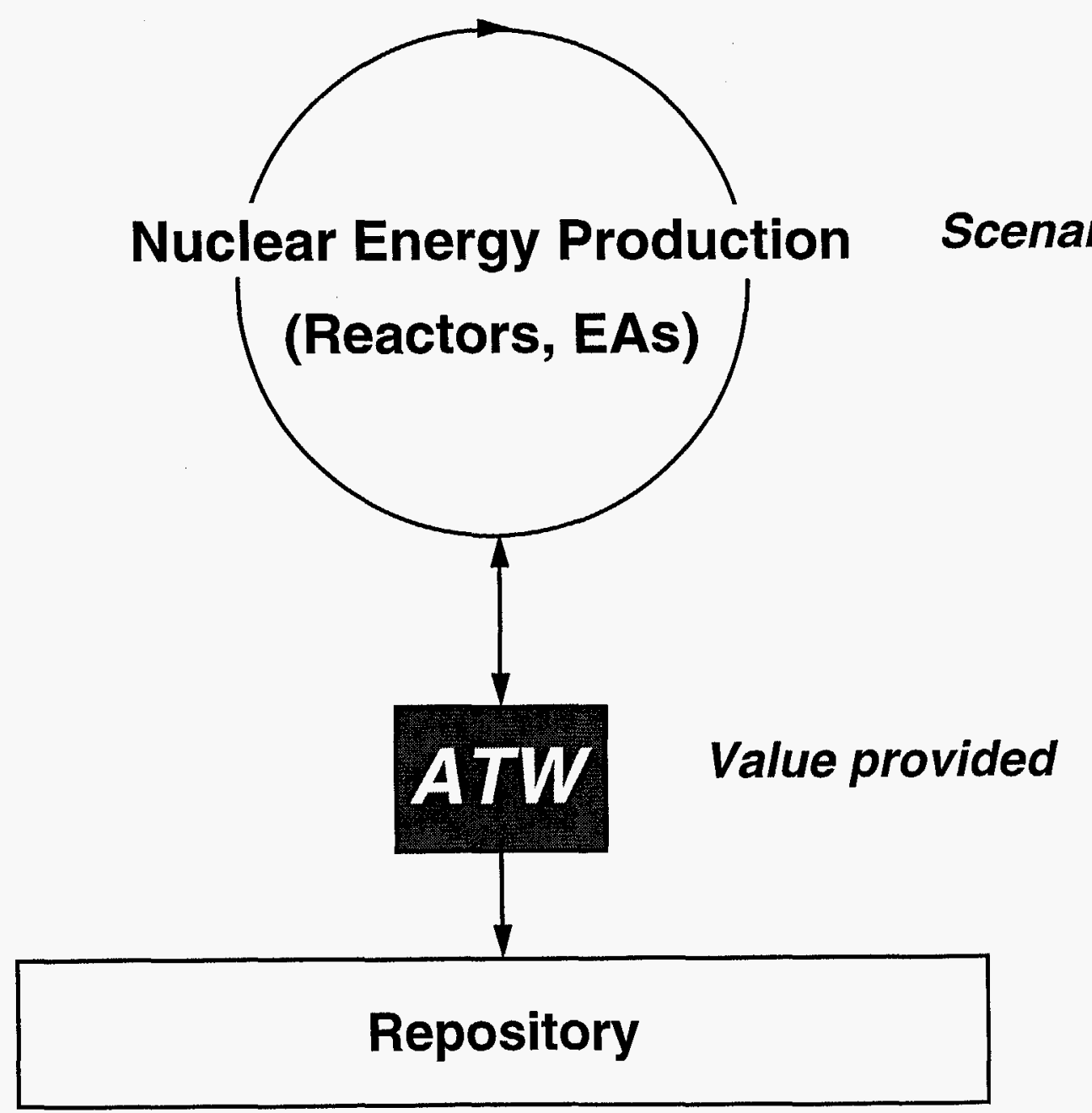




\section{ATW Liquid-Lead Cooled Concept}

Typical Power: 1000 MWt

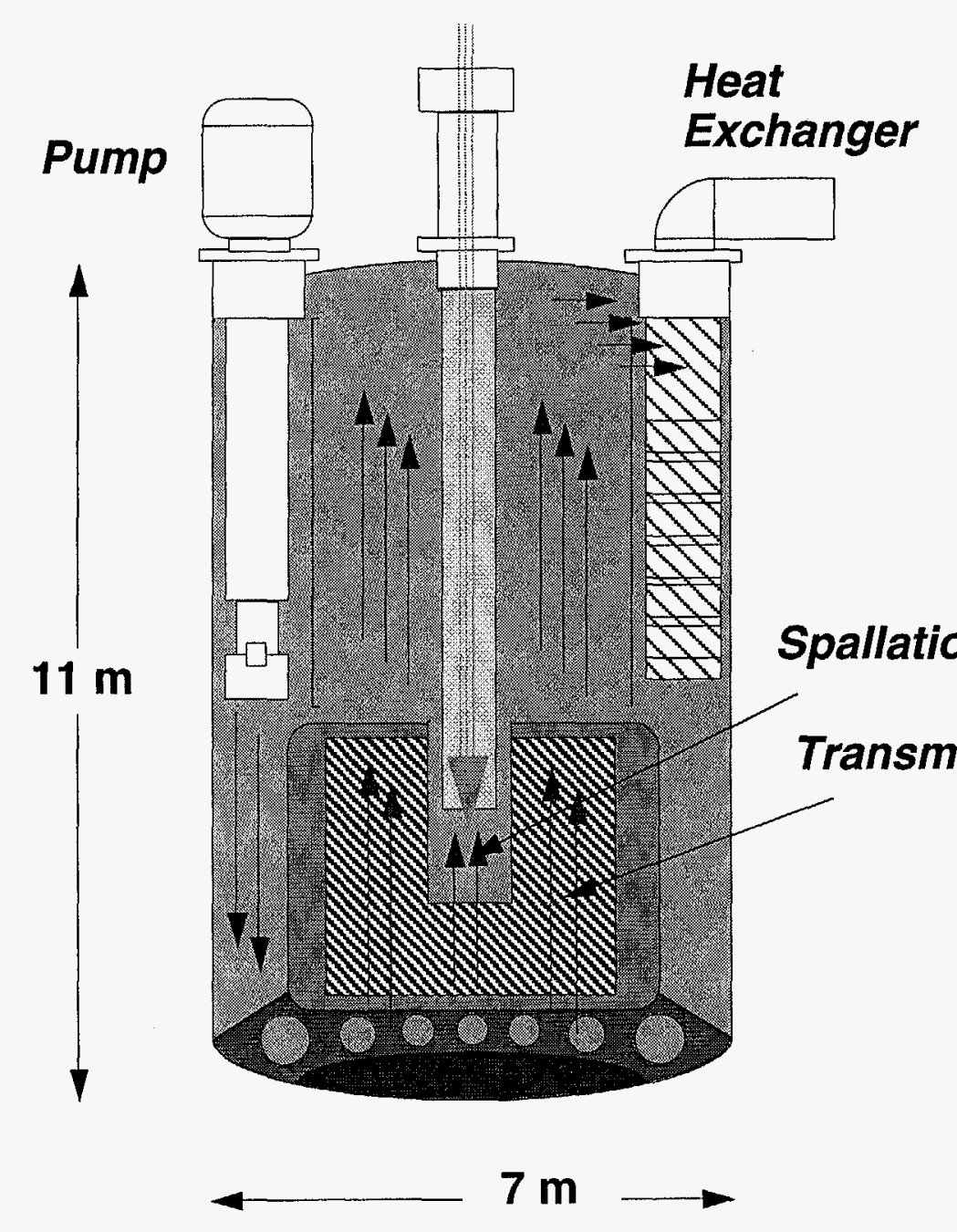

Internal heat exchangers (lead-lead) and pumps.

External pumps and heat exchangers would be more easily serviced. add considerably to pressure drop for lead.

Natural circulation loop for decay heat removal, $15 \%$ heat load capacity

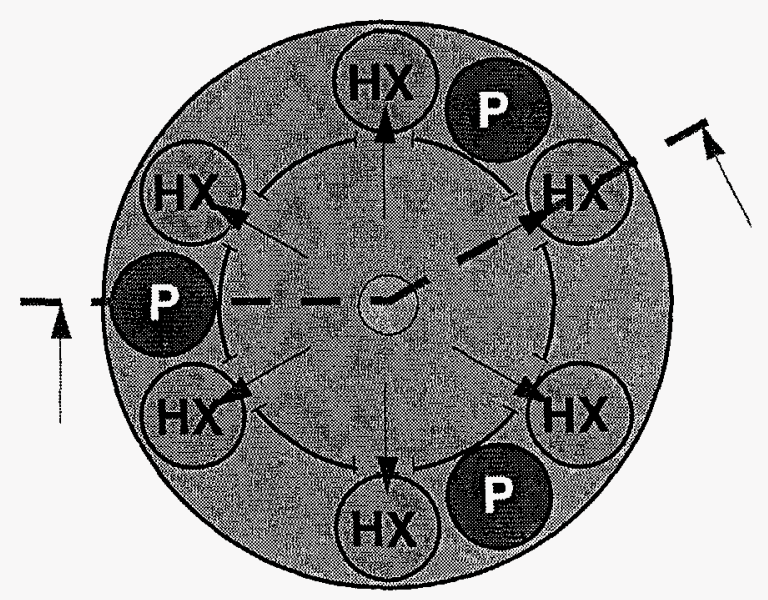

Lead temperature at inlet and outlet $470^{\circ} \mathrm{C}$ and $580^{\circ} \mathrm{C}$ with a maximum lead velocity of $1.4 \mathrm{~m} / \mathrm{s}$.

Maximum main vessel wall temperature is $500^{\circ} \mathrm{C}$.

3 pumps at $5 \mathrm{~m} 3 / \mathrm{s}$ each (80000 GPM), 2.4 bar pressure $5 \mathrm{MW}$ total pump power. 


\section{ATW Primary Choices and Alternatives}

\begin{tabular}{|c|c|c|}
\hline Target & Coolant & Fuel Form \\
\hline Liquid Lead & Liquid Lead & $\begin{array}{l}\text { Liquid Bismuth + } \\
\text { Actinides + Tc }\end{array}$ \\
\hline $\begin{array}{l}\text { Liquid Lead + } \\
\text { Bismuth }\end{array}$ & $\begin{array}{l}\text { Liquid Lead + } \\
\text { Bismuth }\end{array}$ & $\begin{array}{l}\text { Molten Fluorides } \\
\text { Actinides + FpF }\end{array}$ \\
\hline & Liquid Sodium & IFR Metal \\
\hline & & Ceramic \\
\hline
\end{tabular}




\section{LOS ALAMOS I NTERNATIONAL PACIITTY FOR TransuUtations \\ LIFT Provides a Powerful Model for International Cooperation}

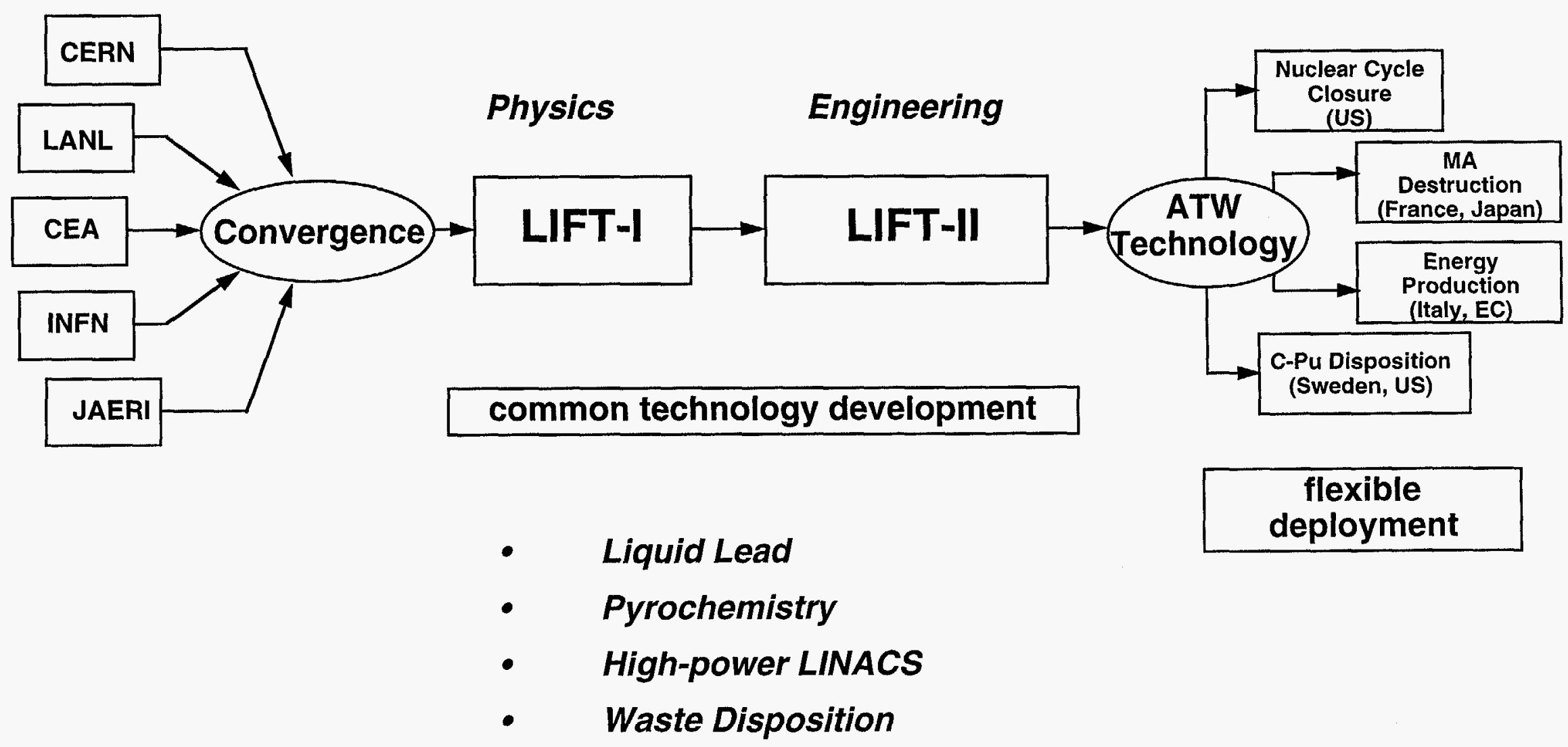




\section{Time Line}

PACILIT FOR

Transhutations

1997 LANL

1999 LANL

2000 LANL

2001 LANL

2006 Europe

2015 US/Europe
Liquid lead loops (LDRD-PD)

Obninsk-IPPE target arrives at LANL

Obninsk-IPPE Target Test

OTT

Liquid Lead Multipurpose Transmutation Exp.

LIFT-I

LIFT- II

Symultaneous deployment of full-scale ATW 
ํㅗㄴ

uo 00t
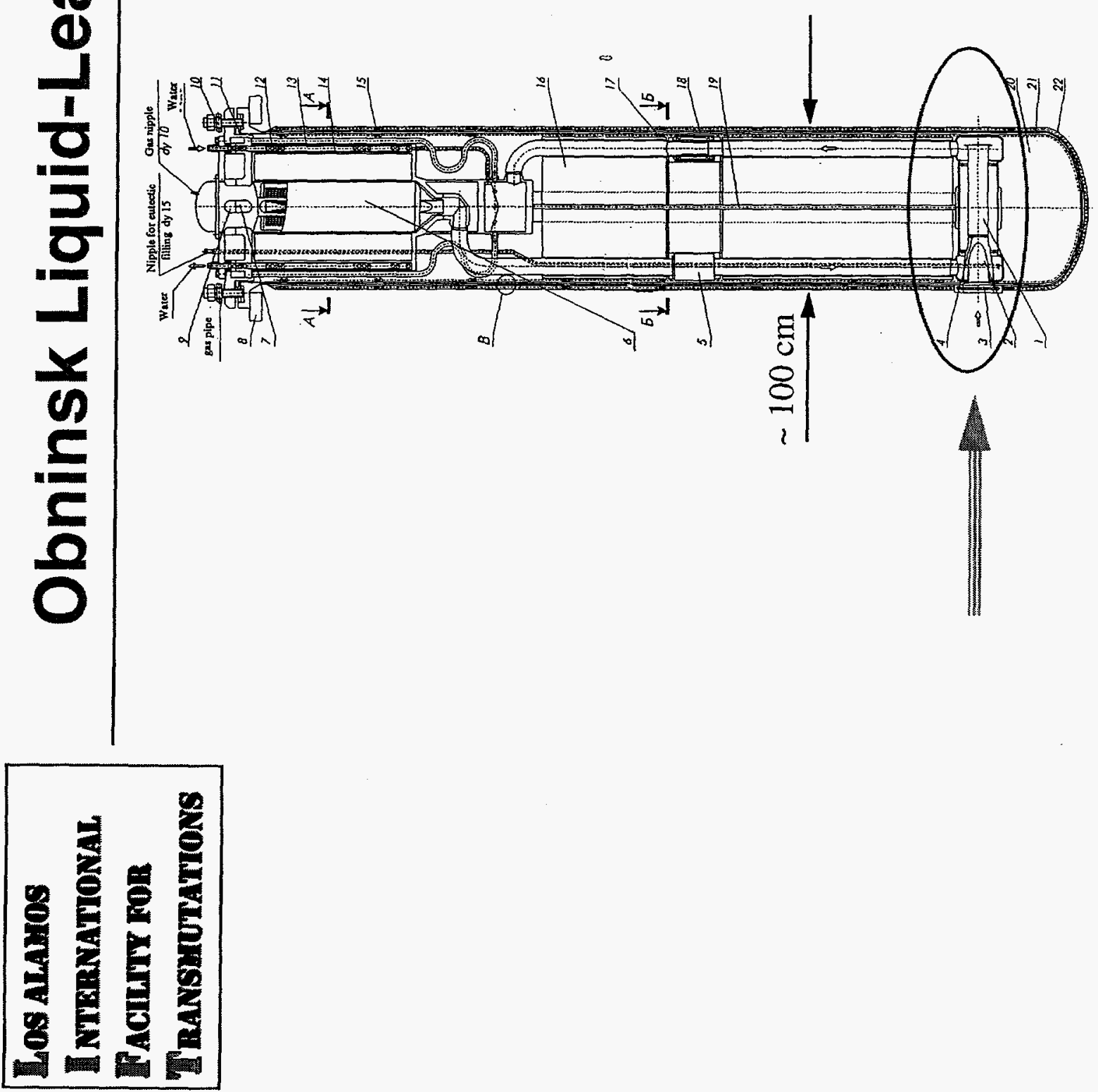


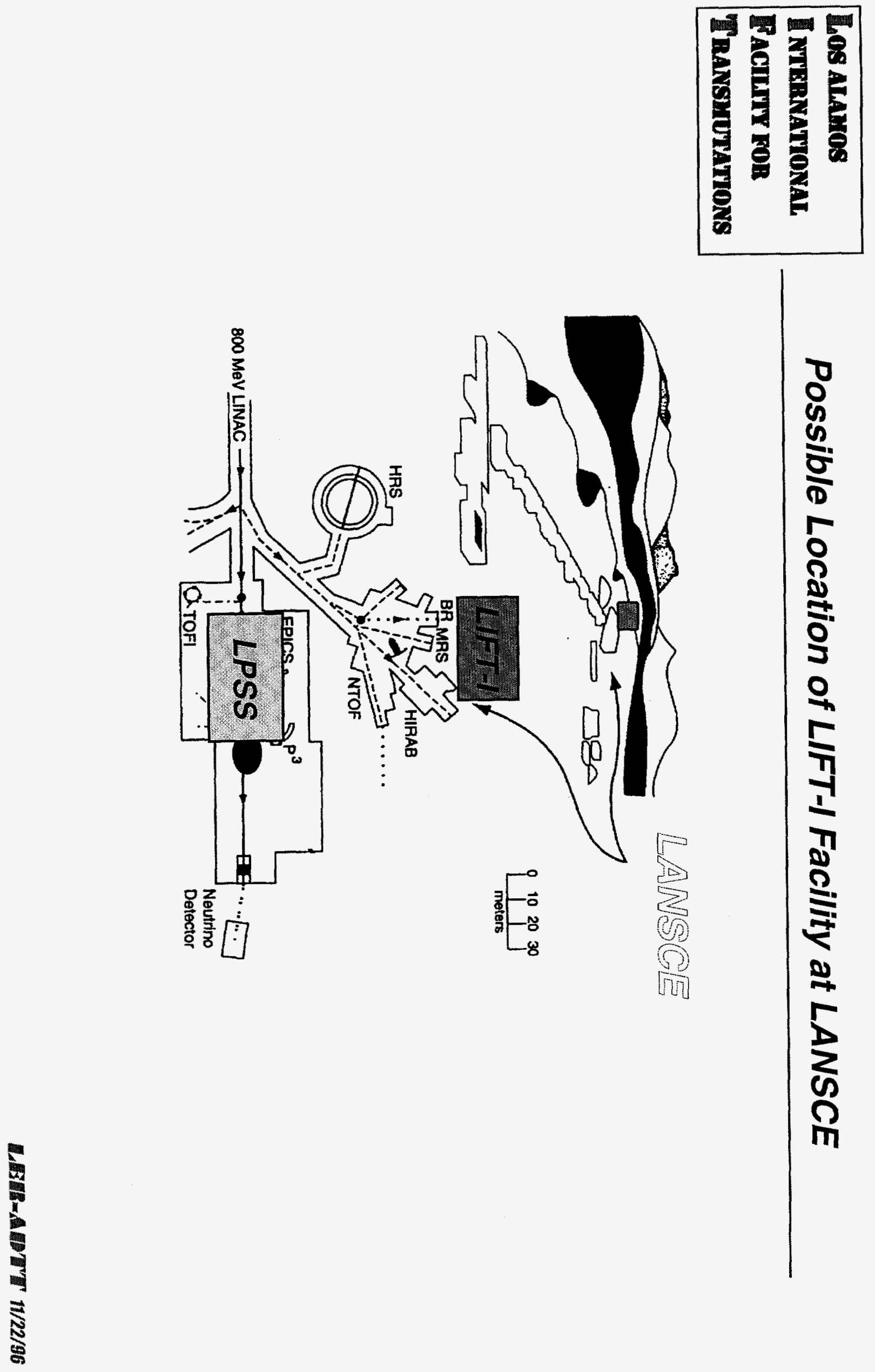

